\title{
The Art and Science of Systems Engineering
}

\author{
Christopher E. Singer, Deputy Director \\ Engineering Directorate \\ NASA Marshall Space Flight Center
}

\begin{abstract}
The National Aeronautics and Space Administration (NASA) was established in 1958, and its Marshall Space Flight Center was founded in 1960, as space-related work was transferred from the Army Ballistic Missile Agency at Redstone Arsenal, where Marshall is located. With this heritage, Marshall contributes almost 50 years of systems engineering experience with humanrated launch vehicles and scientific spacecraft to fulfill NASA's mission of exploration and discovery (fig. 1). ${ }^{1}$ These complex, highly specialized systems have provided vital platforms for expanding the knowledge base about Earth, the solar system, and cosmos; developing new technologies that also benefit life on Earth; and opening new frontiers for America's strategic space goals. ${ }^{2}$ From Mercury and Gemini, to Apollo and the Space Shuttle, Marshall's systems engineering expertise is an unsurpassed foundational competency for NASA and the nation.
\end{abstract}

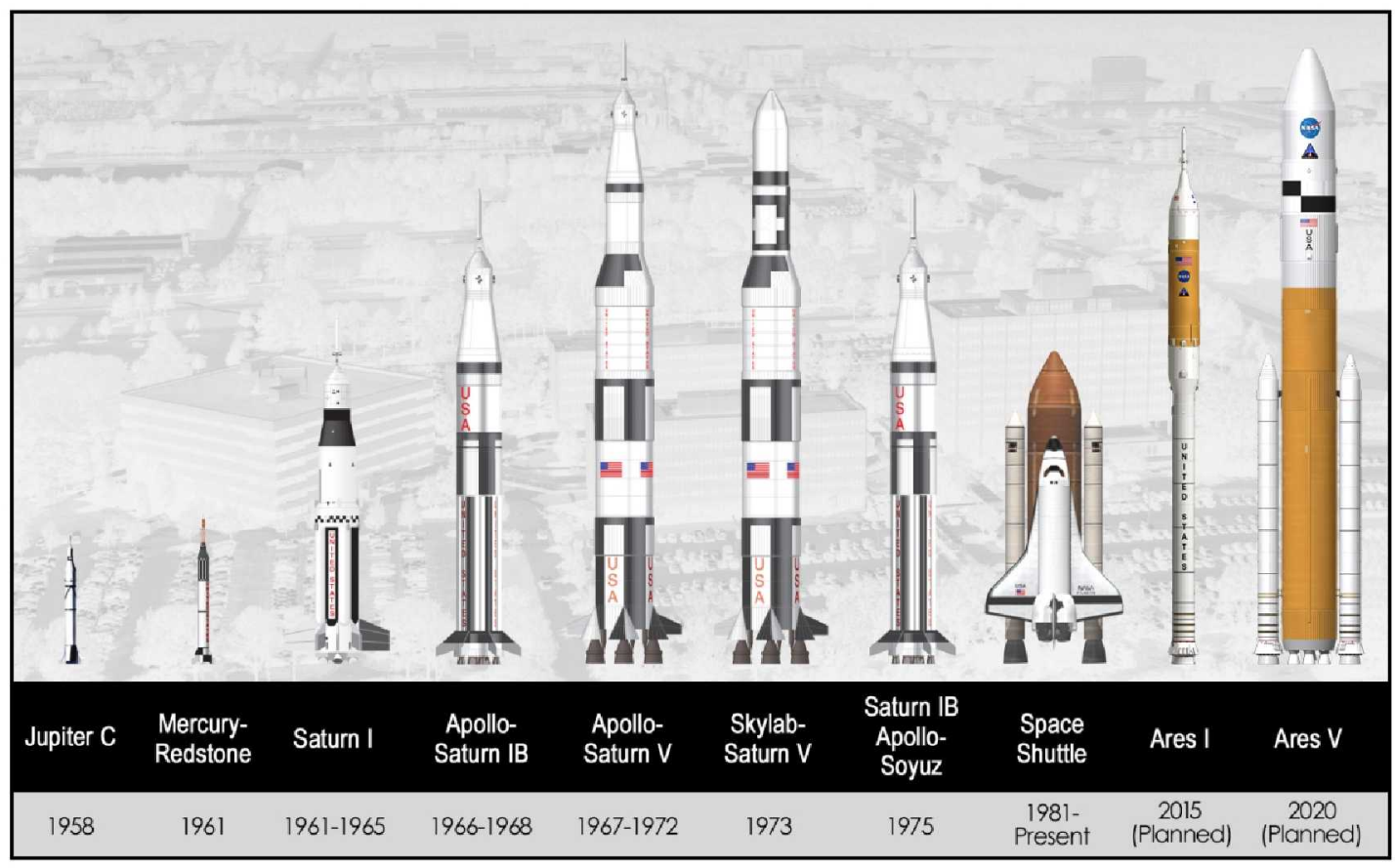

Fig. 1. Marshall builds on almost 50 years of experience with scientific and human-rated launch vehicles. 
Current assignments comprise managing Space Shuttle Propulsion systems (fig. 2); developing environmental control and life support systems and coordinating science operations on the International Space Station (fig. 3); and a number of exploration-related responsibilities. These include managing and performing science missions, such as the Lunar Crater Observation and Sensing Satellite and the Lunar Reconnaissance Orbiter slated to launch for the Moon in April 2009, to developing the Ares I crew launch vehicle upper stage and integrating the vehicle stack in house, as well as designing the Ares $\mathrm{V}$ cargo launch vehicle and contributing to the development of the Altair Lunar Lander and an International Lunar Network with communications nodes and other infrastructure (fig. 4). ${ }^{3}$

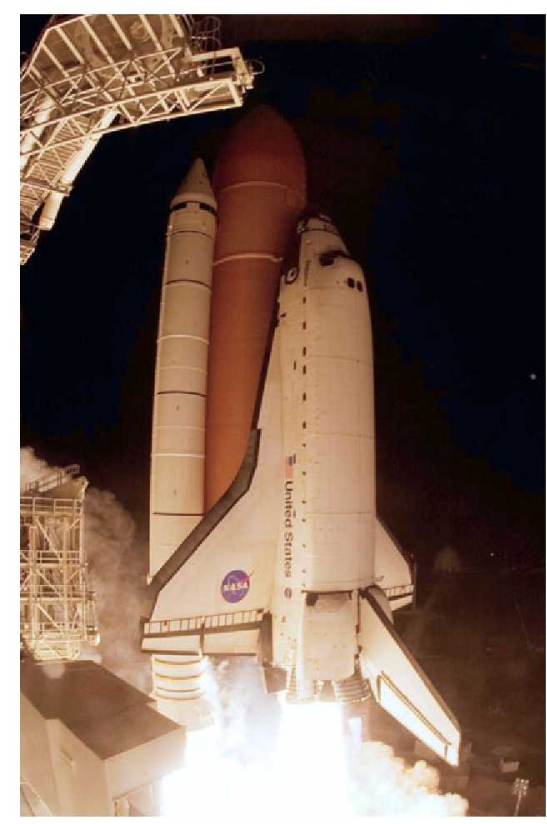

Fig. 2. Marshall developed and manages Space Shuttle propulsion elements (STS-126).

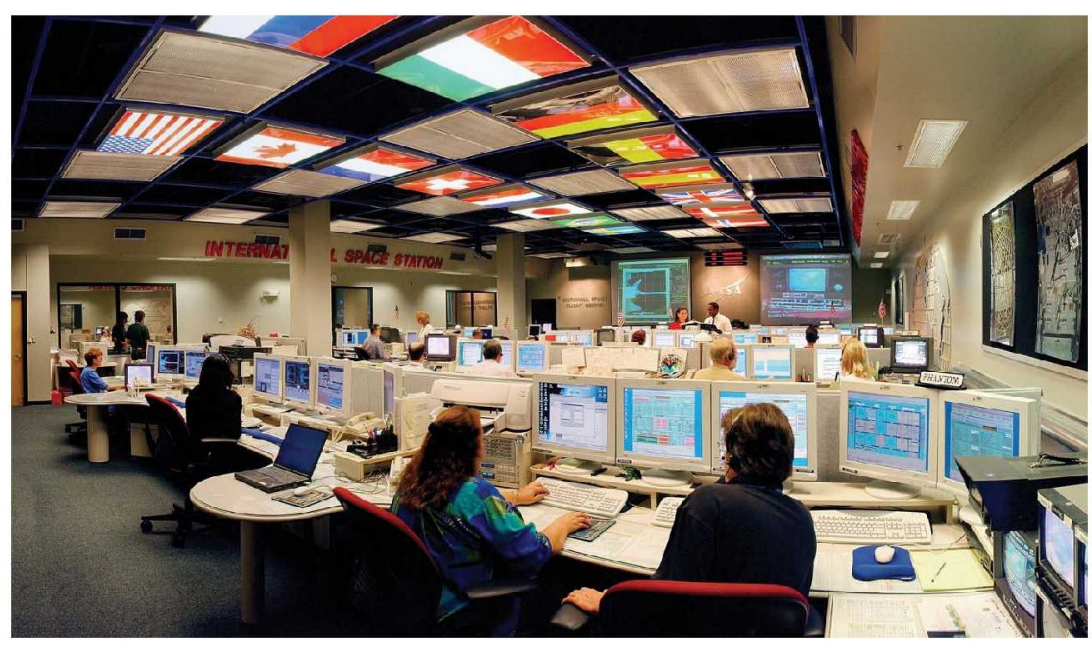

Fig. 3. Marshall's Payload Operations Center manages Space Station science. 


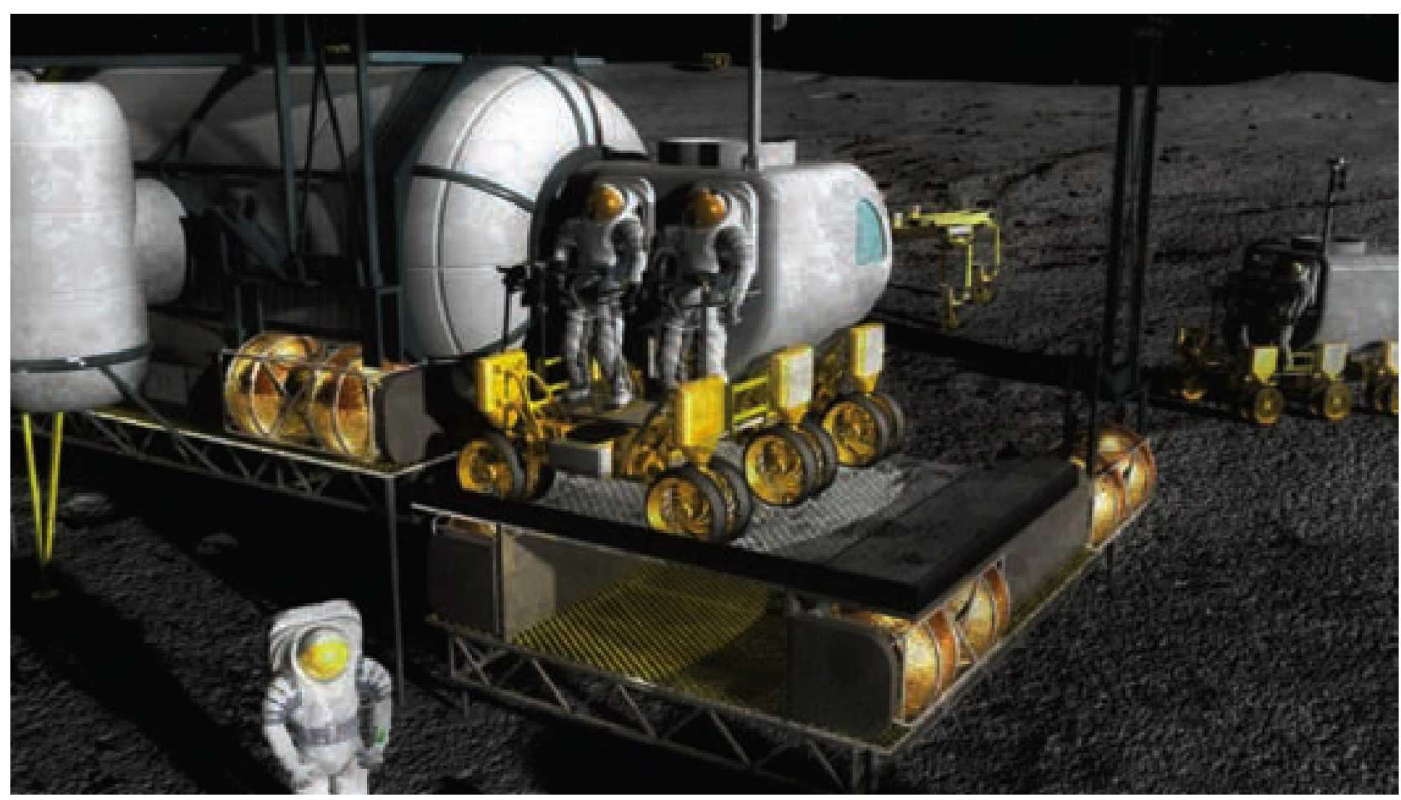

Fig. 4. NASA lunar mission concept.

Fielding an integrated launch vehicle system entails many challenges, not the least of which is the fact that it has been over 30 years since the United States developed a human-rated space transportation system. Over time, whole generations of rocket scientists have passed through the aerospace community without the opportunity to perform such exacting, demanding, and rewarding work. However, with experience leading the design, development, and end-to-end systems engineering and integration of complex launch vehicles, Marshall offers the in-house talent - both junior- and senior-level personnel - to deliver a new national asset to meet the requirements for safe, reliable, and affordable space exploration solutions for the next 50 years. Marshall's Engineering Directorate manages unique aerospace manufacturing and testing facilities and a staff of about 2,600 professionals representing the range of technical disciplines, as well lead systems engineers and a cadre of chief engineers. Recently, 50 engineers fresh out of college were hired and integrated into this workforce. Even so, the age of the average worker at Marshall is 46-years old; this underscores the importance of identifying and nurturing the next generation of systems engineers who will build on the heritage knowledge base to write the next chapter in the history of space. This paper will give an overview of the systems engineering approach being applied to Ares I vehicle integration, discuss NASA's systems engineering philosophy and best practices, and introduce the systems engineering leadership development program being implemented at Marshall to "grow the bench" in this important area. With this as a backdrop, it will provide first-hand observations about the art of systems engineering and why that is critical to this technical competency. 
Realizing the importance of standardizing engineering practices across the board, it is equally important to develop individuals with critical systems thinking capacity, who have the vision and leadership qualities to see beyond the piece parts to the integrated whole. ${ }^{4}$ In this way, systems engineering serves an integrative function (fig. 5) and is the key to delivering a sound system that fully satisfies customer and stakeholder requirements. ${ }^{5}$

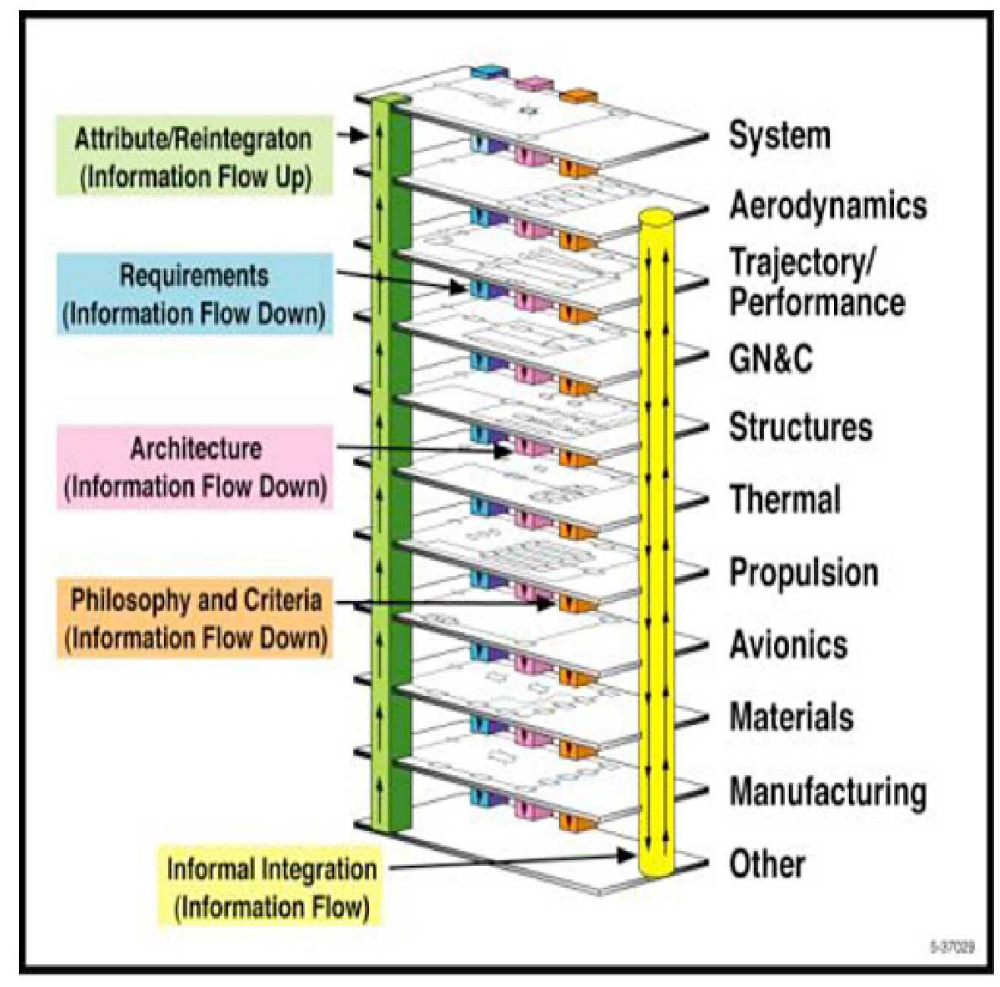

Fig. 5. Systems engineering is an iterative process that bridges functional areas, both horizontally and vertically.

In 2004, the Engineering Directorate reorganized and purposefully did not establish a standalone systems engineering organization, choosing to embed systems engineers throughout functional areas and business units. The culture has, therefore shifted from a stove-piped, disciplined-based view to one where subject matter experts are encouraged, and expected, to explore either side of their specific hardware interface to understand how their piece of the aerospace product is integral to completing a unified whole that functions seamlessly. In this way, compromise, which is the hallmark of delivering engineering solutions, is a natural part of the design process. Within this paradigm, project lifecycle management is the business philosophy that focuses on the end products and serves as an information core platform that unites workers by providing standardized tools designed to increase productivity and accuracy. ${ }^{6}$ Whereas tools have their place, the importance of face-to-face communication and relationships built on a common agenda and trust cannot be overstated. From that perspective, systems engineering is as much an art as a science; this realization is a positive step toward developing a new generation of systems engineers who are well equipped to face the challenges of space exploration. 


\section{AIAA Space 2009}

\section{References}

1. 2006 NASA Strategic Plan, NP-2006-02-423-HQ.

2. U.S. Space Exploration Policy, www.nasa.gov.

3. Global Exploration Strategy, www.nasa.gov.

4. Johnson, Stephen B. The Secret of Apollo: Systems Management in American and European Space Programs, Johns Hopkins University Press, 2002.

5. NASA Systems Engineering Handbook, SP-2007-6105.

6. Grieves, Michael. Project Lifecycle Management: Driving the Next Generation of Lean Thinking, McGraw-Hill, 2006. 